\title{
Suicide Attempts and Childhood Maltreatment Among Street Youth: A Prospective Cohort Study
}

Scott E. Hadland, MD, MPH ${ }^{a, b}$, Evan Wood, MD, PhD ${ }^{c, d}$, Huiru Dong, MSc ${ }^{c}$, Brandon D.L. Marshall, PhD ${ }^{\mathrm{e}}$, Thomas Kerr, PhD ${ }^{c, d}$, Julio S. Montaner, MD ${ }^{\mathrm{c}, \mathrm{d}}$, Kora DeBeck, PhD ${ }^{\mathrm{c}, \mathrm{f}}$

abstract вackground: Although suicide is a known leading cause of death among street youth, few prospective studies have explored childhood experiences as risk factors for future suicide attempt in this population. We examined the risk of attempted suicide in relation to childhood maltreatment among street youth.

METHODS: From September 2005 to November 2013, data were collected from the At Risk Youth Study (ARYS), a prospective cohort of street youth in Vancouver, Canada. Inclusion criteria were age 14 to 26 years, past-month illicit drug use, and street involvement. Participants completed the Childhood Trauma Questionnaire, an instrument measuring self-reported sexual, physical, and emotional abuse and physical and emotional neglect. Suicide attempts were assessed semiannually. Using Cox regression, we examined the association between the 5 types of maltreatment and suicide attempts. RESULTS: Of 660 participants, $68.2 \%$ were male and 24.6\% were Aboriginal. Median age was 21.5 years. The prevalence of moderate to extreme childhood maltreatment ranged from $16.8 \%$ (sexual abuse) to $45.2 \%$ (emotional abuse). Participants contributed 1841 personyears, with suicide attempts reported by 35 (5.3\%) individuals (crude incidence density: 1.9 per 100 person-years; 95\% confidence interval [CI]: 1.4-2.6 per 100 person-years). In adjusted analyses, types of maltreatment associated with suicide attempts included physical abuse (adjusted hazard ratio [HR]: 4.47; 95\% CI: 2.12-9.42), emotional abuse (adjusted HR: 4.92; 95\% CI: 2.11-11.5), and emotional neglect (adjusted HR: 3.08; 95\% CI: 1.05-9.03).

concLusions: Childhood maltreatment is associated with subsequent risk of suicidal behavior among street youth. Suicide prevention efforts should be targeted toward this marginalized population and delivered from a trauma-informed perspective.

WHAT'S KNOWN ON THIS SUBJECT: Street youth demonstrate elevated mortality compared with the general adolescent and young adult population. Suicide is a leading cause of death among street youth. Many street youth have experienced childhood maltreatment, including abuse and neglect.

WHAT THIS STUDY ADDS: In this prospective cohort of street youth, self-reported attempted suicide and history of childhood maltreatment were common. Individuals who experienced childhood physical abuse, emotional abuse, or emotional neglect were at highest risk of attempting suicide.
${ }^{a}$ Division of Adolescent/Young Adult Medicine, Department of Medicine, Boston Children's Hospital, Boston, Massachusetts, ${ }^{b}$ Department of Pediatrics, Harvard Medical School, Boston, Massachusetts; ${ }^{c}$ British Columbia Centre for Excellence in HIV/AIDS, St Paul's Hospital, Vancouver, British Columbia, Canada; ${ }^{d}$ Faculty of Medicine, University of British Columbia, Vancouver, British Columbia, Canada; ${ }^{e}$ Department of Epidemiology, Brown University School of Public Health, Providence, Rhode Island; and ${ }^{f}$ School of Public Policy, Simon Fraser University, Vancouver, British Columbia, Canada

Dr Hadland designed the study, wrote the protocol, conducted the literature review, provided input for statistical analyses, and wrote the first draft of the manuscript; Dr Wood designed the study, wrote the protocol, provided input for statistical analyses, contributed to the first draft of the manuscript, and contributed to the final manuscript; Ms Dong undertook data management and statistical analyses, contributed to the first draft of the manuscript, and contributed to the final manuscript; Dr Marshall designed the study, wrote the protocol, provided input for statistical analyses, and contributed to the final manuscript; Drs Kerr and Montaner designed the study, wrote the protocol, and contributed to the final manuscript; Dr DeBeck designed the study, wrote the protocol, aided with the literature review, provided input for statistical analyses, and contributed to the first draft of the manuscript; and all authors approved the final manuscript as submitted.

www.pediatrics.org/cgi/doi/10.1542/peds.2015-1108

DOI: $10.1542 / p e d s .2015-1108$

Accepted for publication Jun 23, 2015 
Each year, $>10000$ adolescents and young adults die of suicide in the United States, where it is the third leading cause of death among youth aged 15 to 24 years and accounts for $20 \%$ of all deaths in this age group. ${ }^{1}$ In Canada, suicide also accounts for $20 \%$ of all deaths among those $<25$ years old and is the second leading cause of death among adolescents and young adults. ${ }^{2}$ General population-based estimates of suicidal behavior may not accurately reflect rates among hidden youth populations, particularly those who are homeless or street-involved and may be at even higher risk of suicide. Street youth (young people living or working all or part of their time on the street) are a marginalized population with greatly elevated mortality compared with the general youth population. ${ }^{3}$ In one of the only longitudinal studies of mortality among street youth conducted to date, the death rate was 11 times that of the general youth population, and suicide was the single leading cause of death. ${ }^{4}$

Understanding why street youth are at high risk of suicide is critical to developing prevention efforts. Illicit drug use, which is highly prevalent among street youth,5,6 is strongly associated with suicidal ideation and behavior. 4,7-10 Illicit drug use is, in turn, associated with childhood maltreatment, which can include sexual, physical, and emotional abuse and physical and emotional neglect.11,12 Among adult drug users, childhood physical and sexual abuse are determinants of suicidal ideation and attempts. ${ }^{13,14}$ Emotional abuse and physical and emotional neglect have been the subject of less study but are emerging as important risk factors for suicide. ${ }^{15,16} \mathrm{~A}$ history of childhood maltreatment is common among street youth, ${ }^{17}$ but its association with suicide in this vulnerable population has received little study. In addition, the contributions of different types of abuse (sexual, physical, and emotional) and neglect (physical and emotional) to suicide risk have not been systematically examined.

We conducted this prospective cohort study in street youth to examine the risk of attempted suicide in relation to childhood maltreatment. We hypothesized that more severe levels of maltreatment would be associated with an elevated risk of attempting suicide. Consistent with recent data from adults, ${ }^{16}$ we also hypothesized that emotional abuse and neglect would be correlates of risk of attempted suicide.

\section{METHODS}

\section{Study Design}

The At Risk Youth Study (ARYS) is a prospective cohort of street youth in Vancouver, Canada, which has been described previously. ${ }^{18}$ Participants were recruited between September 2005 and November 2013. Inclusion criteria were age 14 to 26 years and past-month use of illicit drugs other than marijuana. (Because marijuana use is highly prevalent among Canadian youth, ${ }^{19}$ inclusion criteria required use of drugs other than marijuana to enroll youth at risk of initiating injection, a primary study outcome.) Eligible youth were street-involved, defined as having been without housing in the preceding 6 months, or, if not homeless, having accessed services for street-involved youth during that time. 6,20,21

Recruitment occurred during daytime and nighttime in neighborhoods where street youth were known to congregate, with snowball sampling to recruit additional participants. Informed consent was obtained, and baseline and semiannual follow-up interviews were completed at a storefront location in downtown Vancouver. Participants were listed in study records by name, but identities were safeguarded by institutional review board-approved study protocols. ${ }^{18}$ As part of consent procedures, participants were advised verbally and in writing of the legal duty to report abuse of persons aged $<19$ years in British Columbia and were assured of their right to refuse to answer questions on this or any other topic. Participants were remunerated 20 Canadian dollars at baseline and follow-up visits. The ARYS was approved by the University of British Columbia and Providence Health Care Research Ethics Board.

\section{Measures}

Participants underwent an interviewer-administered questionnaire capturing sociodemographic characteristics, drug use, and sexual behaviors. The questionnaire included the Center for Epidemiologic Studies Depression (CES-D) scale, a validated instrument with a standardized cutoff of $>21$ indicating high levels of depressive symptoms. ${ }^{22,23}$ At baseline and follow-up visits occurring semiannually, participants also completed a nurse-administered interview with questions on past-6month suicide attempts. Emergency referral services were available for participants with active suicidal ideation or suspected ongoing abuse or neglect.

The primary outcome of interest was the response to the question, "In the last 6 months, have you attempted suicide?" We also ascertained lifetime suicidal ideation with the question, "Have you ever seriously thought about taking your own life?" Nurse interviewers were trained to differentiate intentional suicide attempts from accidental drug overdoses. The primary exposure was self-reported childhood maltreatment based on responses to the Childhood Trauma Questionnaire (CTQ), which participants completed at baseline. The CTQ is a validated 25-item measure that has been successfully used among street-involved youth, 
with subscales detecting 5 types of maltreatment: (1) sexual abuse, (2) physical abuse, (3) emotional abuse, (4) physical neglect, and (5) emotional neglect. ${ }^{24-26}$

The CTQ provides a separate score for each type of maltreatment based on 5-point Likert responses ranging from 1 ("never true") to 5 ("very often true"). All questions began with the prompt, "When I was growing up...," to specifically address experiences from childhood. Sample questions included, “...I was punished with a belt, a board, a cord, or some other hard object" (physical abuse) and "...People in my family said hurtful or insulting things to me" (emotional abuse). ${ }^{26}$ We used validated standard cutoffs for each of the 5 CTQ subscales: "no or minimal" maltreatment (score of 5-8), "low to moderate" (score of 9-12), "moderate to severe" (score of 13-15), and "severe to extreme" (score $>15$ ). 26,27

Other measured characteristics included the following: age, gender, Aboriginal ancestry, sexual identity (lesbian, gay, bisexual, or transgender), current enrollment in or previous completion of high school, past-6-month homelessness, use of street-based outreach services (outreach worker, street nurse, health van, home care worker/nurse, safe injection facility, or youth drop-in center), daily alcohol use, injection drug use, overdose, sex work (trading sex for money/drugs/shelter/ clothing), and CES-D score $>21$.

\section{Sample}

Although the ARYS continued open enrollment beyond the study period for the present analysis, the sample included here was restricted to the 660 (66.0\%) participants of 1002 individuals recruited who had returned for $\geq 1$ follow-up visit by November 2013. Those who returned for follow-up were similar to those who did not with regard to key study variables, including past-6-month suicide attempts, CTQ subscale scores, and all other characteristics $(P>.05$ for all).

\section{Statistical Analyses}

We calculated the proportion of youth reporting a suicide attempt during the study period and, on the basis of the person-time method, calculated the crude incidence density of attempted suicide. We next compared those who reported a suicide attempt during follow-up with those who did not with regard to baseline characteristics. Analyses used the Pearson $\chi^{2}$ test and Fisher's exact test (for cell counts $<5$ ) for categorical variables and the Wilcoxon test for continuous variables. We then compared those with and without a suicide attempt according to each type of childhood maltreatment using the Cochran-Mantel-Haenszel test for ordinal data.

Next, using the Kaplan-Meier method, we compared the cumulative incidence of attempted suicide according to CTQ scores of 5 to 12 ("none to moderate") versus scores $>12$ ("moderate to extreme") for each type of maltreatment. (Although these terminologies both included "moderate" severity of abuse, score groupings were mutually exclusive and score ranges were based on standardized cutoffs of the CTQ. ${ }^{26,27}$ Scores were dichotomized because of the high prevalence of low-moderate childhood maltreatment. ${ }^{16}$ ) Participants who attempted suicide were right-censored at the time of their first attempt; the remaining participants were right-censored at the time of their last follow-up visit. We compared survival distributions using the log-rank test.

Then, using Cox proportional hazards regression, we examined associations between the 5 types of maltreatment and suicide attempts in 5 separate models. We also generated a combined analysis in which we entered all types of maltreatment into the same model to identify the independent contributions of each. All multivariable analyses adjusted for age and gender on the basis of their known association with risk of suicide among youth. ${ }^{28,29}$ In addition, because previous studies of mortality among street youth have shown that death from drug overdose may be difficult to differentiate from suicide, ${ }^{4}$ we also adjusted for past-6-month injection drug use and drug overdose. Variables were time-updated.

Because the outcome of interest, suicide attempt, might have been associated with loss to follow-up (ie, informative censoring), we conducted separate analyses in which we generated models using inverse probability of censoring weights (IPCW) for comparison. ${ }^{30}$ IPCW analyses reweighted the sample such that the contribution of participants who did not return for follow-up was increased, thus generating effect estimates that would have been calculated if every participant had provided full follow-up. As described elsewhere, ${ }^{31,32}$ these analyses modeled the probability of remaining uncensored according to CTQ score and other study variables with the use of pooled logistic regression. We also linked to provincial registries to obtain information on deaths among participants lost to follow-up.

Finally, to examine the role of depressive symptoms as an intermediate in the pathway between childhood maltreatment and suicide attempts, we used the Sobel method to determine whether CES-D score mediated the relationship between each type of maltreatment and suicide attempts. ${ }^{33}$

Analyses were performed by using SAS version 9.3 (SAS Institute, Cary, NC). All $P$ values were 2 -sided and tests were considered significant at $P<.05$.

\section{RESULTS}

Of 660 eligible participants, 68.2\% were male, $24.6 \%$ were Aboriginal, 

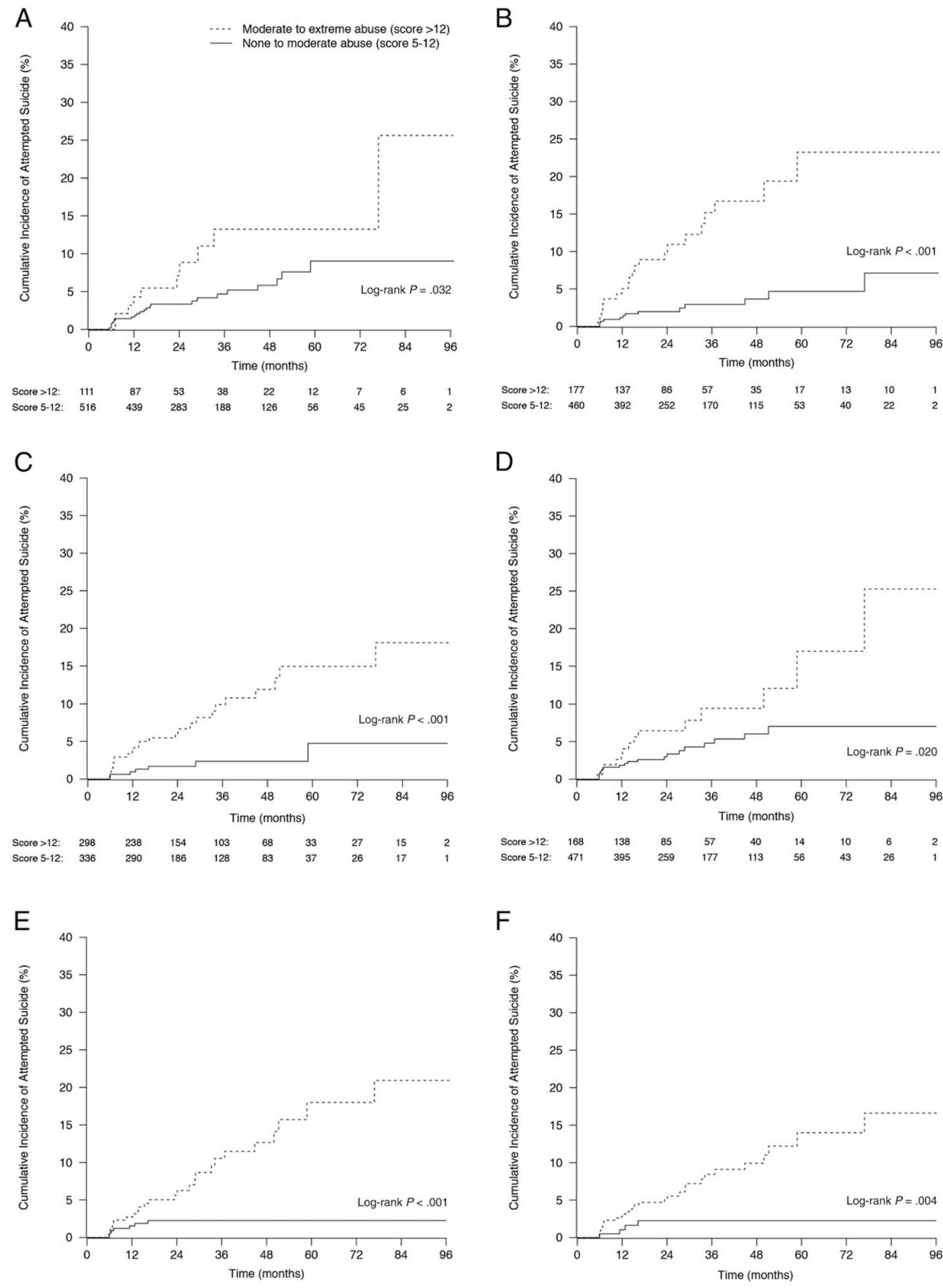

$\mathrm{F}$
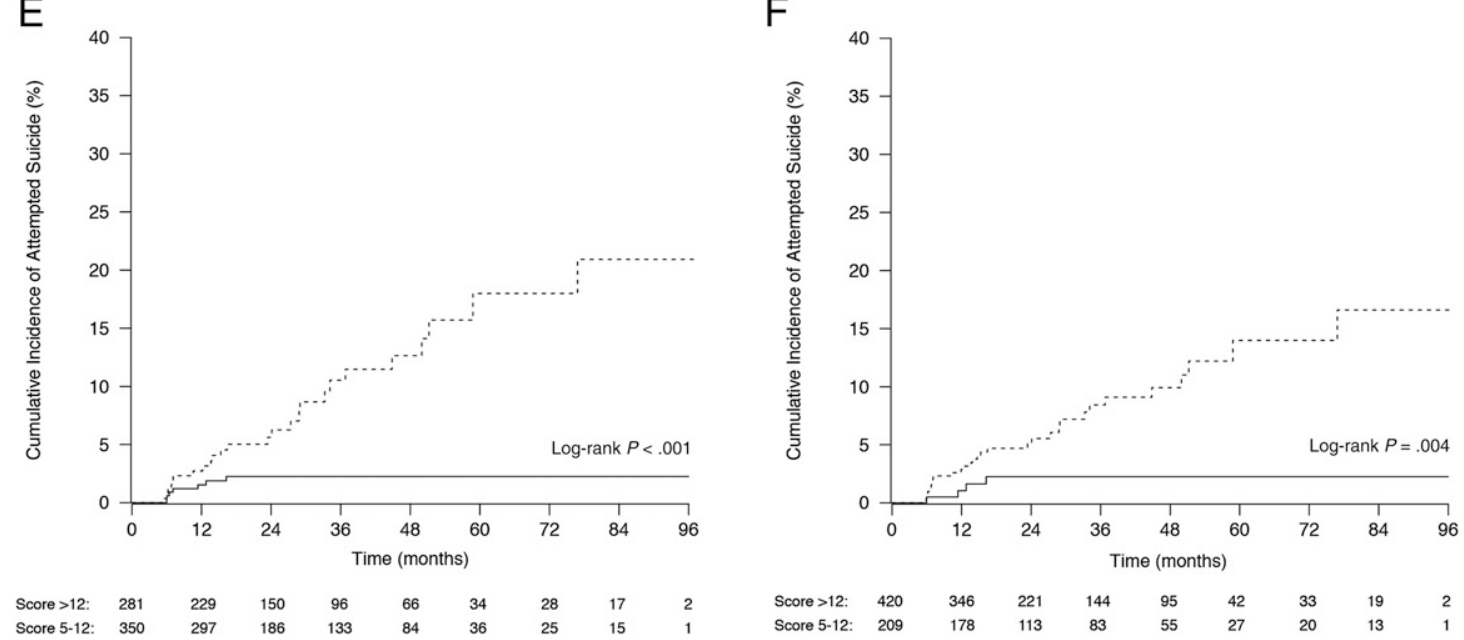

$\begin{array}{llllllllll}\text { Score }>12: & 420 & 346 & 221 & 144 & 95 & 42 & 33 & 19 & 2 \\ \text { Score 5-12: } & 209 & 178 & 113 & 83 & 55 & 27 & 20 & 13 & 1\end{array}$

FIGURE 1

Cumulative incidence of attempting suicide according to severity of sexual abuse (A), physical abuse (B), emotional abuse (C), physical neglect (D), emotional neglect (E), and any type of abuse/neglect (F): ARYS (Vancouver, British Columbia; 2005-2013). $N=660$. Although both groupings listed ("moderate to severe abuse," "none to moderate abuse") include "moderate" levels of abuse, categories used standardized cutoffs of the CTQ and score ranges were mutually exclusive. $^{26,27}$ Categories were collapsed with "none to moderate" (score of 5-12) incorporating "none to minimal" (score of 5-8) combined with "low to moderate" (score of 9-12), and "moderate to severe" (score >12) incorporating "moderate to severe" (score of 13-15) combined with "severe to extreme" (score $>15$ ). Where totals do not add to 660 at time zero, values are missing because the participant elected not to complete the CTQ at the baseline visit. 
TABLE 1 Baseline Characteristics of 660 Street Youth Who Did and Did Not Attempt Suicide During Study Follow-up: ARYS (Vancouver, British Columbia; 2005-2013)

\begin{tabular}{|c|c|c|c|}
\hline \multirow[t]{2}{*}{ Characteristic } & \multicolumn{2}{|c|}{ Suicide Attempt ${ }^{\mathrm{a}}$} & \multirow[b]{2}{*}{$P$} \\
\hline & Yes $(n=35)$ & No $(n=625)$ & \\
\hline Median age, y (IQR) & $21.5(19.8-23.1)$ & $21.8(19.8-23.6)$ & .518 \\
\hline Gender & & & .747 \\
\hline Male & $23(65.7)$ & $427(68.3)$ & \\
\hline Female & $12(34.3)$ & $198(31.7)$ & \\
\hline Aboriginal ancestry & & & .812 \\
\hline Yes & $8(22.9)$ & $154(24.6)$ & \\
\hline No & $27(77.1)$ & $471(75.4)$ & \\
\hline Lesbian/gay/bisexual/transgender & & & .508 \\
\hline Yes & $7(20.0)$ & $98(15.7)$ & \\
\hline No & $28(80.0)$ & $523(83.7)$ & \\
\hline High school education ${ }^{\mathrm{b}}$ & & & .277 \\
\hline Yes & $9(25.7)$ & $215(34.4)$ & \\
\hline No & $26(74.3)$ & $405(64.8)$ & \\
\hline Homeless $^{\mathrm{C}}$ & & & .140 \\
\hline Yes & $29(82.9)$ & $443(70.9)$ & \\
\hline No & $6(17.1)$ & $178(28.5)$ & \\
\hline Use of outreach services ${ }^{\mathrm{c}, \mathrm{d}}$ & & & .252 \\
\hline Yes & $31(88.6)$ & $505(80.8)$ & \\
\hline No & $4(11.4)$ & $120(19.2)$ & \\
\hline Daily alcohol use ${ }^{c}$ & & & .639 \\
\hline Yes & $7(20.0)$ & $105(16.8)$ & \\
\hline No & $28(80.0)$ & $515(82.4)$ & \\
\hline Injection drug use ${ }^{\mathrm{c}}$ & & & .445 \\
\hline Yes & $8(22.9)$ & $180(28.8)$ & \\
\hline No & $27(77.1)$ & $444(71.0)$ & \\
\hline Drug overdose ${ }^{c}$ & & & .105 \\
\hline Yes & $7(20.0)$ & $68(10.9)$ & \\
\hline No & $28(80.0)$ & $552(88.3)$ & \\
\hline Sex work ${ }^{\mathrm{C}}$ & & & .554 \\
\hline Yes & $4(11.4)$ & $57(9.1)$ & \\
\hline No & $31(88.6)$ & 568 (90.9) & \\
\hline CES-D ${ }^{\mathrm{e}}$ score $>21$ & & & .037 \\
\hline Yes & $19(54.3)$ & $213(34.1)$ & \\
\hline No & $16(45.7)$ & $367(58.7)$ & \\
\hline
\end{tabular}

Data are presented as $n$ (\%) unless otherwise indicated; $N=660$. Characteristics were reported at the time of study enrollment.

a Cells do not uniformly add up to column totals due to missing values.

b Previous completion of or current enrollment in high school.

c During the preceding 6 months.

d Street-based outreach services include outreach worker, street nurse, health van, home care worker/nurse, safe injection facility, or youth drop-in center.

e CES-D scale using a standardized cutoff of $>21$.

and $15.9 \%$ identified as lesbian, gay, bisexual, or transgender, with a median age of 22 years (interquartile range [IQR]: $20-24$ years) at baseline. A total of $320(48.5 \%)$ participants reported having ever lived in an orphanage, a foster home, or a group home. Lifetime suicidal ideation was reported by 259 (39.2\%) individuals.

Participants contributed 1841 person-years of total follow-up (median follow-up per participant: 26 months; IQR: 16-48 months; median visits: 4; IQR: 2-7).

Provincial records revealed that 7 deaths were observed among those after their last follow-up visit; causes of death included 1 suicide and 1 overdose, and the cause was unclear in 5 cases. Suicide attempts were reported by 35 (5.3\%) individuals during follow-up, resulting in a crude incidence density of 1.9 per 100 person-years $(95 \%$ confidence interval: $1.4-2.6$ per 100 personyears). Six participants reported $>1$ suicide attempt (median: 2; maximum: 4), resulting in a total of 44 attempts for the 35 participants reporting an attempt during followup. As shown in Table 1, individuals who reported a suicide attempt were more likely to have a CES-D score $>21$ at baseline but otherwise did not differ significantly on other characteristics.

Although every participant in the sample completed all or part of the CTQ, scores were missing for the sexual abuse subscale from $33(5.0 \%)$ participants, for the physical abuse subscale from 23 (3.5\%), for the emotional abuse subscale from 26 (3.9\%), for the physical neglect subscale from 21 (3.2\%), and for the emotional neglect subscale from 29 (4.4\%). In addition, the CES-D scale was not fully completed by 45 (6.8\%) participants. For both instruments, respondents did not differ from nonrespondents in terms of suicide attempts and all other variables $(P>.05$ for all). CTQ respondents did not differ from nonrespondents with regard to CES-D score $(P>.05)$. Each of the CTQ subscales was correlated with one another $(P<.001$ for all pairs of subscales), with correlation coefficients ranging from 0.21 (sexual abuse and emotional neglect) to 0.49 (physical abuse and emotional abuse).

As shown in Table 2, increasing severity of childhood maltreatment was associated with attempting suicide for all types of maltreatment except for sexual abuse. The cumulative incidence of suicide attempts was significantly associated with all types of maltreatment examined separately (Fig $1 \mathrm{~A}, \mathrm{~B}, \mathrm{C}, \mathrm{D}$, and E) and together (Fig 1F). As shown in Table 3, which lists crude and adjusted hazard ratios according to type of maltreatment, we observed a significantly elevated risk of suicide attempts among those reporting "moderate to extreme" physical abuse, emotional abuse, and emotional neglect but not among 
TABLE 2 Associations of Childhood Maltreatment With Suicide Attempts During Follow-up: ARYS (Vancouver, British Columbia; 2005-2013)

\begin{tabular}{|c|c|c|c|c|}
\hline \multirow[t]{2}{*}{ Type of Maltreatment (CTQ Subscale Score) ${ }^{a}$} & \multirow[t]{2}{*}{ Total $^{\mathrm{b}}(N=660), n(\%)$} & \multicolumn{2}{|c|}{ Suicide Attempt, $n$ (\%) } & \multirow[b]{2}{*}{$P^{\mathrm{c}}$} \\
\hline & & Yes $(n=35)$ & No $(n=625)$ & \\
\hline Sexual abuse & & & & .062 \\
\hline None to minimal (5-8) & $476(72.1)$ & $22(62.9)$ & $454(72.6)$ & \\
\hline Low to moderate $(9-12)$ & $40(6.1)$ & $1(2.9)$ & $39(6.2)$ & \\
\hline Moderate to severe (13-15) & $31(4.7)$ & $2(5.7)$ & $29(4.6)$ & \\
\hline Severe to extreme $(>15)$ & $80(12.1)$ & $8(22.9)$ & $72(11.5)$ & \\
\hline Physical abuse & & & & $<.001$ \\
\hline None to minimal (5-8) & $345(52.3)$ & $9(25.7)$ & $336(53.8)$ & \\
\hline Low to moderate $(9-12)$ & $115(17.4)$ & $4(11.4)$ & $111(17.8)$ & \\
\hline Moderate to severe (13-15) & $56(8.5)$ & $8(22.9)$ & $48(7.7)$ & \\
\hline Severe to extreme $(>15)$ & $121(18.3)$ & $13(37.1)$ & $108(17.3)$ & \\
\hline Emotional abuse & & & & $<.001$ \\
\hline None to minimal (5-8) & $208(31.5)$ & $3(8.6)$ & $205(32.8)$ & \\
\hline Low to moderate $(9-12)$ & $128(19.4)$ & $4(11.4)$ & $124(19.8)$ & \\
\hline Moderate to severe $(13-15)$ & $98(14.9)$ & 8 (22.9) & $90(14.4)$ & \\
\hline Severe to extreme $(>15)$ & $200(30.3)$ & $17(48.6)$ & $183(29.3)$ & \\
\hline Physical neglect & & & & .013 \\
\hline None to minimal (5-8) & $300(45.5)$ & $10(28.6)$ & $290(46.4)$ & \\
\hline Low to moderate (9-12) & $171(25.9)$ & $9(25.7)$ & $162(25.9)$ & \\
\hline Moderate to severe (13-15) & $87(13.2)$ & $6(17.1)$ & $81(13.0)$ & \\
\hline Severe to extreme $(>15)$ & $81(12.3)$ & $8(22.9)$ & $73(11.7)$ & \\
\hline Emotional neglect & & & & $<.001$ \\
\hline None to minimal (5-8) & $179(27.1)$ & $1(2.9)$ & $178(28.5)$ & \\
\hline Low to moderate $(9-12)$ & $171(25.9)$ & $6(17.1)$ & $165(26.4)$ & \\
\hline Moderate to severe (13-15) & $104(15.8)$ & $11(31.4)$ & $93(14.9)$ & \\
\hline Severe to extreme $(>15)$ & $177(26.8)$ & $14(40.0)$ & $163(26.1)$ & \\
\hline
\end{tabular}

those reporting "moderate to extreme" sexual abuse or physical neglect. Effect sizes were comparable in IPCW models, which reweighted the sample to account for informative censoring (ie, to account for the possibility that those lost to follow-up were at greatest risk of suicide). In the combined model, which included all types of maltreatment in the same model, only physical abuse retained a statistically significant hazard ratio.

CES-D score did not appear to mediate the relationship between statistically significant forms of maltreatment and suicide attempts.

Sobel test statistics for mediation by CES-D scores $>21$ were as follows: physical abuse, 1.75 $(P=.080)$; emotional abuse, 1.77 $(P=.077)$; and emotional neglect, $1.55(P=.122)$.

\section{DISCUSSION}

In this prospective cohort study in street youth, we observed a high incidence of self-reported attempted suicide, with $>1$ in 20 youth reporting an attempt over the 7-year study period. History of childhood maltreatment was strongly associated with risk of attempted suicide, with youth who reported previous physical abuse, emotional abuse, or emotional neglect 3 to nearly 5 times as likely to report attempting suicide. Among the 5 types of self-reported childhood maltreatment examined in a combined model, only physical abuse retained an independent effect on risk of attempted suicide.

To date, most studies on suicidality and childhood maltreatment have focused on adult drug users in clinicbased samples and have used a cross-sectional design. ${ }^{13,14,34}$ Our study is novel in examining a longitudinal cohort of communityrecruited street youth. Although we observed a high frequency of attempted suicide in our sample, studies reveal that attempted suicide is alarmingly common throughout the general adolescent population. The 2013 Youth Risk Behavior Surveillance Study of US high school students estimated the past-year prevalence of attempted suicide to be $8 \%$ (girls: $11 \%$; boys: $5 \%$ ). ${ }^{35} \mathrm{In}$ British Columbia, the estimated pastyear prevalence of attempted suicide in the general adolescent population in 2013 was $6 \%$ (girls: $9 \%$; boys: $3 \%$ ). Despite the similar prevalence of attempted suicide among street youth and the general adolescent population, the most common methods used among street youth are especially deadly and include wrist slitting, intentional drug overdose, and hanging. ${ }^{36}$

Lifetime suicidal ideation was reported by $39 \%$ of participants in our study, a prevalence $>3$ times that reported by the National Comorbidity Survey of adolescents in the United States. ${ }^{37}$ This elevated prevalence in part reflects the higher median age in our study sample, because suicidality generally increases as adolescence progresses to early adulthood. ${ }^{1,2,28}$ In any case, estimates of lifetime suicidal ideation among adults in the general population are one-third that found in the street youth in our study, and factors such as childhood maltreatment are likely critical contributors. 38,39

Our results extend those from crosssectional studies in clinic-based adult drug users. ${ }^{13,14,34}$ Our results also build on findings from a recent longitudinal study of communityrecruited adult drug users in Vancouver showing elevated risk of attempted suicide after childhood maltreatment. ${ }^{16}$ What is less clear among these studies is which types of childhood maltreatment confer the greatest risk. In studies examining multiple types of maltreatment, 
TABLE 3 Crude and Adjusted Hazard Ratios for Attempting Suicide: ARYS (Vancouver, British Columbia; 2005-2013)

\begin{tabular}{|c|c|c|c|c|}
\hline \multirow[t]{2}{*}{ Type of Maltreatment ${ }^{a}$} & \multirow[t]{2}{*}{ Crude Hazard Ratio (95\% Cl) } & \multicolumn{3}{|c|}{ Adjusted Hazard Ratio ${ }^{\mathrm{b}}$ (95\% Cl) } \\
\hline & & Standard Model & IPCW Model & Combined Model \\
\hline Sexual abuse & $1.71(0.80-3.64)$ & $1.63(0.70-3.78)$ & $1.43(0.58-3.55)$ & $1.00(0.43-2.34)$ \\
\hline Physical abuse & $4.62(2.20-9.71)$ & $4.47(2.12-9.42)$ & $5.09(2.27-11.4)$ & $2.74(1.03-7.28)$ \\
\hline Emotional abuse & $4.66(1.93-11.2)$ & $4.92(2.11-11.5)$ & $4.45(1.82-10.9)$ & $2.52(0.88-7.25)$ \\
\hline Physical neglect & $1.84(0.89-3.82)$ & $1.70(0.80-3.59)$ & $1.88(0.84-4.19)$ & $0.76(0.36-1.60)$ \\
\hline Emotional neglect & $3.36(1.28-8.81)$ & $3.08(1.05-9.03)$ & $2.95(1.02-8.54)$ & $1.42(0.40-5.06)$ \\
\hline \multicolumn{5}{|c|}{$\begin{array}{l}N=660 . \\
\text { a Categories used standardized cutoffs of the CTQ and were collapsed with "none to moderate" (score of 5-12; reference group) incorporating "none to minimal" (score of 5-8) combined } \\
\text { with "low to moderate" (score of 9-12), and "moderate to severe" (score >12; comparison) incorporating "moderate to severe" (score of 13-15) combined with "severe to extreme" } \\
\left(\text { score >15). }{ }^{26,27}\right.\end{array}$} \\
\hline
\end{tabular}

results often suggest that sexual abuse shows the strongest association with suicidality. ${ }^{15,34}$ In our sample, sexual abuse was associated with increased cumulative incidence of attempted suicide but was not significant in Cox regression models. Sexual abuse was the least common form of childhood maltreatment reported in our study, although we cannot exclude that participants may have felt uncomfortable disclosing such experiences.

Nonetheless, our study builds on previous studies by including less studied forms such as emotional abuse and physical and emotional neglect. Although less researched, emotional abuse has shown a strong association with suicidality in studies in which it has been examined. ${ }^{16,34}$ In our study, emotional abuse was reported to have co-occurred in nearly half of all participants also reporting physical abuse, and emotional neglect was similarly comorbid. Because physical abuse was the only type of maltreatment to maintain an independent effect in a combined model, some of the harm of emotional abuse and neglect may be due to physical abuse that youth experience simultaneously.

The underlying mechanisms linking childhood maltreatment to suicide require further study. Although our data did not support a mediating role for depressive symptoms, childhood maltreatment and suicidality are both linked to depression, bipolar disorder, anxiety disorders, and posttraumatic stress disorder. ${ }^{37,40}$ Such psychiatric conditions, many of which are prevalent among street youth, may result from childhood maltreatment and subsequently predispose to suicidality. 3,6,41 Prolonged street involvement is also likely detrimental to the mental health of youth. ${ }^{42}$ Homelessness is an independent predictor of substance use. ${ }^{43}$ Both homelessness and substance use are harmful yet common aspects of street life, $, 44,45$ and both are associated with suicidality. ${ }^{46,47}$ Qualitative research shows that homelessness, substance use, and adverse childhood experiences converge on feelings of hopelessness and of being "trapped," which contribute to suicide among street youth. ${ }^{48}$

Regardless of mechanism, previous work suggests that to deliver effective, trauma-informed suicide treatment and prevention to street youth, providers should understand that the impact of childhood maltreatment is far-reaching and influences child and adolescent development and coping strategies. ${ }^{49,50}$ Street youth providers should ensure a physically and emotionally safe treatment environment to avoid retraumatization and, in delivering services, decrease hierarchical approaches and empower youth to collaborate in their own care. ${ }^{49,51}$

Limitations of our study include the reliance on self-report, which may have been subject to recall bias. Participants may have been reluctant to disclose traumatic experiences, suicide attempts, or other risk behaviors. Moreover, distinguishing suicide from drug overdose may be difficult, particularly because overdose is a common method of suicide. . $^{4,8,13}$ To account for this possibility, we trained nurse interviewers to distinguish between overdose and suicide and adjusted for cooccurring injection drug use and recent overdose in our analyses. In addition, we studied childhood maltreatment and did not ascertain ongoing, street-based abuse, which may have contributed to the risk of attempted suicide. ${ }^{52}$ Conversely, we cannot rule out that ongoing contact with research staff and referrals to mental health services for high-risk participants reduced suicidality.

\section{CONCLUSIONS}

Our findings reveal an elevated risk of suicide attempts among street youth with a history of childhood maltreatment compared with those 
without this history. These results build on preexisting research by specifically examining the harms of other, less studied forms of maltreatment, including emotional abuse and physical and emotional neglect. Additional studies are needed to show the mechanistic pathways and to identify protective factors. Nonetheless, the high prevalence of self-reported childhood maltreatment among street youth in this setting demonstrates the importance of delivering suicide prevention services from a trauma-informed perspective.

\section{ACKNOWLEDGMENTS}

We thank the study participants for their contribution to the research, as well as current and past researchers and staff. We specifically thank Ms Sabina Dobrer, Mr Cody Callon, Ms Jennifer Matthews, Ms Deborah Graham, Mr Peter Vann, Mr Steve Kain, Ms Kristie Starr, Ms Tricia Collingham, and Ms Carmen Rock for their research and administrative assistance. We also appreciate the support of Drs S. Jean Emans, Elizabeth R. Woods, and Sarah A.B. Pitts and the Division of
Adolescent/Young Adult Medicine at Boston Children's Hospital.

ABBREVIATIONS

ARYS: At Risk Youth Study

CES-D: Center for Epidemiologic Studies Depression

CTQ: Childhood Trauma Questionnaire

IPCW: inverse probability of censoring weights

IQR: interquartile range

Address correspondence to Kora DeBeck, PhD, British Columbia Centre for Excellence in HIV/AIDS, St Paul's Hospital, 608-1081 Burrard St, Vancouver, BC, Canada V6Z 1Y6. E-mail: uhri-kd@cfenet.ubc.ca

PEDIATRICS (ISSN Numbers: Print, 0031-4005; Online, 1098-4275)

Copyright (C) 2015 by the American Academy of Pediatrics

FINANCIAL DISCLOSURE: As an HIV physician, Dr Montaner has received limited unrestricted funding, paid to his institution, from Abbvie, Bristol-Myers Squibb, Gilead Sciences, Janssen, Merck, and ViiV Healthcare; the other authors have indicated they have no financial relationships relevant to this article to disclose.

FUNDING: Supported by National Institutes of Health (NIH)/National Institute on Drug Abuse (NIDA) grants R01DA028532 and U01DA038886 as well as the Canadian Institutes of Health Research (CIHR) operating grant MOP-102742. In addition, Dr Hadland is supported by the Maternal and Child Health/Health Resources and Services Administration Leadership Education in Adolescent Health Training Program (T71 MC00009) and by a National Research Service Award from NIH/National Institute of Child Health and Human Development (1T32 HD075727). Dr Wood is supported by the Canada Research Chairs program through a Tier 1 Canada Research Chair in Inner City Medicine. Dr Marshall is supported by NIH/NIDA grant R03 DA037770. Dr Montaner is supported by the British Columbia Ministry of Health and by NIH/NIDA grant R01DA036307. Dr DeBeck is supported by a Michael Smith Foundation for Health Research/St Paul's Hospital/Providence Health Care Career Scholar Award and a CIHR New Investigator Award. Funded by the National Institutes of Health (NIH)

POTENTIAL CONFLICT OF INTEREST: The authors have indicated they have no potential conflicts of interest to disclose.

\section{REFERENCES}

1. Johnson NB, Hayes LD, Brown K, Hoo EC, Ethier KA; Centers for Disease Control and Prevention. CDC National Health Report: leading causes of morbidity and mortality and associated behavioral risk and protective factors-United States, 2005-2013. MMWR Surveill Summ. 2014; 63(suppl 4):3-27

2. Statistics Canada. The 10 leading causes of death, 2011. Available at: www.statcan. gc.ca/pub/82-625-x/2014001/article/11896eng.htm. Accessed December 3, 2014

3. Public Health Agency of Canada. Street youth in Canada: findings from the enhanced surveillance of Canadian street youth, 1999-2003. 2006. Available at: www.phac-aspc.gc.ca/std-mts/ reports_06/pdf/street_youth_e.pdf. Accessed December 3, 2014

4. Roy E, Haley N, Leclerc P, Sochanski B, Boudreau JF, Boivin JF. Mortality in a cohort of street youth in Montreal. JAMA. 2004;292(5):569-574

5. Greene JM, Ennett ST, Ringwalt CL. Substance use among runaway and homeless youth in three national samples. Am J Public Health. 1997;87 (2) 229-235

6. Boivin JF, Roy E, Haley N, Galbaud du Fort $\mathrm{G}$. The health of street youth: a Canadian perspective. Can J Public Health. 2005; 96(6):432-437

7. Marshall BDL, Galea S, Wood E, Kerr T. Injection methamphetamine use is associated with an increased risk of attempted suicide: a prospective cohort study. Drug Alcohol Depend. 2011; 119(1-2):134-137

8. Bohnert AS, Roeder K, Ilgen MA. Unintentional overdose and suicide among substance users: a review of overlap and risk factors. Drug Alcohol Depend. 2010;110(3):183-192

9. Wilcox HC, Conner KR, Caine ED. Association of alcohol and drug use disorders and completed suicide: an empirical review of cohort studies. Drug Alcohol Depend. 2004;76(suppl):S11-S19

10. Rohde P, Noell J, Ochs L, Seeley JR. Depression, suicidal ideation and STDrelated risk in homeless older adolescents. J Adolesc. 2001;24(4): 447-460

11. Ompad DC, Ikeda RM, Shah N, et al Childhood sexual abuse and age at initiation of injection drug use. Am J Public Health. 2005;95(4):703-709

12. Dube SR, Felitti VJ, Dong M, Chapman DP, Giles WH, Anda RF. Childhood abuse, neglect, and household dysfunction and the risk of illicit drug use: the adverse 
childhood experiences study. Pediatrics. 2003;111(3):564-572

13. Bohnert ASB, Roeder KM, Ilgen MA. Suicide attempts and overdoses among adults entering addictions treatment: comparing correlates in a U.S. National Study. Drug Alcohol Depend. 2011; 119(1-2):106-112

14. Roy A. Risk factors for attempting suicide in heroin addicts. Suicide Life Threat Behav. 2010;40(4):416-420

15. Brown J, Cohen P, Johnson JG, Smailes EM. Childhood abuse and neglect: specificity of effects on adolescent and young adult depression and suicidality. $J$ Am Acad Child Adolesc Psychiatry. 1999; 38(12):1490-1496

16. Marshall BDL, Galea S, Wood E, Kerr T. Longitudinal associations between types of childhood trauma and suicidal behavior among substance users: a cohort study. Am J Public Health. 2013; 103(9):e69-e75

17. Feldmann J, Middleman AB. Homeless adolescents: common clinical concerns. Semin Pediatr Infect Dis. 2003;14(1):6-11

18. Wood E, Stoltz JA, Montaner JSG, Kerr T. Evaluating methamphetamine use and risks of injection initiation among street youth: the ARYS study. Harm Reduct J. 2006;3:18

19. Fischer B, Rehm J, Hall W. Cannabis use in Canada: the need for a 'public health' approach. Can J Public Health. 2009; 100(2):101-103

20. DeMatteo D, Major C, Block B, et al. Toronto street youth and HIV/AIDS: prevalence, demographics, and risks. J Adolesc Health. 1999;25(5):358-366

21. Kerr T, Marshall BD, Miller C, et al. Injection drug use among street-involved youth in a Canadian setting. BMC Public Health. 2009;9(171):171

22. Radloff LS. The use of the Center for Epidemiologic Studies Depression scale in adolescents and young adults. J Youth Adolesc. 1991;20(2):149-166

23. Radloff LS. The CES-D scale: a self report depression scale for research in the general population. Appl Psychol Meas. 1977;1(3):385-401

24. Scher CD, Stein MB, Asmundson GJ, McCreary DR, Forde DR. The Childhood Trauma Questionnaire in a community sample: psychometric properties and normative data. J Trauma Stress. 2001; 14(4):843-857

25. Forde DR, Baron SW, Scher CD, Stein MB. Factor structure and reliability of the childhood trauma questionnaire and prevalence estimates of trauma for male and female street youth. $J$ Interpers Violence. 2012;27(2):364-379

26. Bernstein DP, Fink L. Childhood Trauma Questionnaire: A Retrospective Selfreport Manual. San Antonio, TX: Psychological Corporation; 1998

27. Bernstein DP, Stein JA, Newcomb MD, et al. Development and validation of a brief screening version of the Childhood Trauma Questionnaire. Child Abuse Negl. 2003;27(2):169-190

28. Hallfors DD, Waller MW, Ford CA, Halpern CT, Brodish PH, Iritani B. Adolescent depression and suicide risk: association with sex and drug behavior. Am J Prev Med. 2004;27(3):224-231

29. Skinner R, McFaull S. Suicide among children and adolescents in Canada: trends and sex differences, 1980-2008. CMAJ. 2012;184(9):1029-1034

30. Robins JM. Information recovery and bias adjustment in proportional hazards regression analysis of randomized trials using surrogate markers. Proceedings of the Biopharmaceutical Section, American Statistical Association. 1993: 22-33

31. Cain LE, Cole SR. Inverse probability-ofcensoring weights for the correction of time-varying noncompliance in the effect of randomized highly active antiretroviral therapy on incident AIDS or death. Stat Med. 2009;28(12): 1725-1738

32. Robins JM, Finkelstein DM. Correcting for noncompliance and dependent censoring in an AIDS clinical trial with inverse probability of censoring weighted (IPCW) log-rank tests. Biometrics. 2000;56(3):779-788

33. Sobel ME. Asymptotic intervals for indirect effects in structural equation models. In: Leinhardt S, ed. Sociological Methodology. San Francisco, CA: JosseyBass Publishers; 1982:290-312

34. Lloyd JJ, Ricketts EP, Havens JR, et al. The relationship between lifetime abuse and suicidal ideation in a sample of injection drug users. J Psychoactive Drugs. 2007; 39(2):159-166
35. Kann L, Kinchen S, Shanklin SL, et al; Centers for Disease Control and Prevention. Youth risk behavior surveillance-United States, 2013. MMWR Surveill Summ. 2014;63(suppl 4):1-168

36. Yoder KA. Comparing suicide attempters, suicide ideators, and nonsuicidal homeless and runaway adolescents. Suicide Life Threat Behav. 1999;29(1): 25-36

37. Nock MK, Green JG, Hwang I, et al. Prevalence, correlates, and treatment of lifetime suicidal behavior among adolescents: results from the National Comorbidity Survey Replication Adolescent Supplement. JAMA Psychiatry. 2013;70(3):300-310

38. Kessler RC, Berglund P, Borges G, Nock $M$, Wang PS. Trends in suicide ideation, plans, gestures, and attempts in the United States, 1990-1992 to 2001-2003. JAMA. 2005;293(20):2487-2495

39. Kessler RC, Borges G, Walters EE. Prevalence of and risk factors for lifetime suicide attempts in the National Comorbidity Survey. Arch Gen Psychiatry. 1999;56(7):617-626

40. Green JG, McLaughlin KA, Berglund PA, et al. Childhood adversities and adult psychiatric disorders in the national comorbidity survey replication I: associations with first onset of DSM-IV disorders. Arch Gen Psychiatry. 2010; 67(2):113-123

41. Kelly K, Caputo T. Health and street/ homeless youth. J Health Psychol. 2007; 12(5):726-736

42. Karabanow J. Getting off the street: exploring the processes of young people's street exits. Am Behav Sci. 2008; 51(6):772-788

43. Feng C, DeBeck K, Kerr T, Mathias S, Montaner J, Wood E. Homelessness independently predicts injection drug use initiation among street-involved youth in a Canadian setting. J Adolesc Health. 2013;52(4):499-501

44. Fazel S, Khosla V, Doll H, Geddes J. The prevalence of mental disorders among the homeless in Western countries: systematic review and meta-regression analysis. PLoS Med. 2008;5(12):e225

45. Hwang SW. Homelessness and health. CMAJ. 2001;164(2):229-233

46. Eynan R, Langley J, Tolomiczenko G, et al. The association between homelessness 
and suicidal ideation and behaviors: results of a cross-sectional survey. Suicide Life Threat Behav. 2002;32(4): 418-427

47. Greene JM, Ringwalt CL. Youth and familial substance use's association with suicide attempts among runaway and homeless youth. Subst Use Misuse. 1996; 31(8):1041-1058

48. Kidd SA. "The walls were closing in, and we were trapped": a qualitative analysis of street youth suicide. Youth Soc. 2004; 36(1):30-55
49. McKenzie-Mohr S, Coates J, McLeod H. Responding to the needs of youth who are homeless: calling for politicized trauma-informed intervention. Child Youth Serv Rev. 2012;34(1):136-143

50. Prescott L, Soares P, Konnath K, Bassuk E. A Long Journey Home: A Guide for Creating Trauma-Informed Services for Mothers and Children Experiencing Homelessness. Rockville, MD: Substance Abuse and Mental Health Services Administration 2008. Available at: http:// homeless.samhsa.gov/ResourceFiles/
ALongJourneyHome.pdf. Accessed May 28, 2015

51. Elliott DE, Bjelajac P, Fallot RD, Markoff LS, Reed BG. Trauma-informed or trauma-denied: principles and implementation of trauma-informed services for women. J Community Psychol. 2005;33(4):461-477

52. Goodman L, Saxe L, Harvey M. Homelessness as psychological trauma: broadening perspectives. Am Psychol. 1991;46(11):1219-1225

A WONDERFUL 800 $^{\text {th }}$ BIRTHDAY: I was recently vacationing in England with my daughter. I took her to some of my favorite museums, including the British Museum, the Tate Modern, the Victoria and Albert, and the British Library. The British Library has a fantastic collection, including Shakespeare folios, Da Vinci notebooks, a Gutenberg bible, and an ancient copy of Beowulf. However, I really wanted her to see the Magna Carta.

As reported in The New York Times (Europe: June 14, 2015), the Magna Carta is 800 years old this year. Revered by many, the Magna Carta is often considered the foundation of English and American law. Interestingly, views of the importance of the Magna Carta in 2015 are radically different from that of 1215 or otheryears, and quite different in England and the United States. The Magna Carta was a negotiated peace treaty between rebellious barons angered by King John's tax levies, blackmail, and legal manipulations. Written in Latin (the language of the Church but not the court or the people), the document mostly deals with husbandry, land issues, and feudal financial arrangements including inheritance. The agreement was between the elite of medieval society and the King, but did not specifically include commoners. While King John did affix his seal in agreement at Runnymede, he immediately appealed to the Pope to annul the agreement, which the Pope did two months later. Edited and reissued several times over the next 100 years, the versions we most associate with the Magna Carta were most likely written in 1225 and 1297. Interestingly, almost no part of the charter was actually honored in the years after 1215. Only four of the original 64 provisions are still part of English law. Nonetheless, a core principle was recorded - that the King could not imprison people whenever he wanted for whatever reason he wanted. While it took centuries for due process to become part of enforceable English law, the concepts written in 1215 are still applicable today. Earlier this year, a provision in the charter was cited in a US Supreme Court decision on judicial integrity.

While I am not sure my daughter was particularly impressed by the document itself, she and I could both appreciate its historical significance.

Noted by WVR, MD 